\title{
Un instrumento para evaluar la calidad lingüístico-discursiva de textos disciplinares producidos por estudiantes de Derecho ${ }^{1}$
}

An instrument to evaluate the linguistic-discursive quality of disciplinary texts produced by law students

\section{Paulina Meza \\ Universidad de La Serena \\ Chile}

\section{Felipe González Catalán}

Pontificia Universidad Católica de Valparaíso Chile

\section{(c) $\bigodot_{\mathrm{BY}} \bigodot_{\mathrm{ND}}$}

Paulina Meza: Universidad de La Serena (Instituto de Investigación Multidisciplinar en Ciencia y Tecnología), Chile. | E-mail: pmeza@userena.cl

Felipe González Catalán: Escuela de Derecho, Facultad de Derecho, Pontificia Universidad Católica de Valparaíso. | E-mail: felipe.gonzalez.c@pucv.cl 


\section{Resumen}

En esta investigación proponemos como objetivo generar un instrumento que permita evaluar la calidad lingüístico-discursiva de textos disciplinares producidos por estudiantes de Derecho. Para ello, desarrollamos un estudio cualitativo con diseño fenomenológico, a fin de proponer una rúbrica que permita orientar la enseñanza y evaluación de la escritura en la formación en Derecho. El proceso de creación del instrumento incluyó entrevistas a los profesores de la carrera mencionada, lo que permitió realizar un levantamiento de las propiedades lingüístico-discursivas que debiesen poseer los textos especializados de la disciplina en cuestión y los aspectos que debiesen evaluar los docentes disciplinares en dichos textos. Una vez diseñada, sometimos la rúbrica a diversos procedimientos de validación, luego de los cuales quedó, finalmente, constituida por 16 dimensiones y 4 macrodimensiones: situación comunicativa, comunicación del conocimiento jurídico, estructura global y oracional y normativa. La versión final del instrumento obtuvo un acuerdo considerable tanto para la validez de contenido $(K=0,73)$ como para la confiabilidad intercodificador $(K=0,62)$. El hecho de contar con un instrumento validado será de gran utilidad para los distintos actores involucrados en el proceso de formación de abogados, pues permitirá obtener información más precisa respecto del nivel de adquisición de la competencia escritural en Derecho. Así también, permitirá definir estrategias de intervención adecuadas a las necesidades de los estudiantes y proveer herramientas que posibiliten el desarrollo de una didáctica de la escritura en Derecho.

Palabras clave: alfabetización académica; evaluación de la escritura; Derecho; rúbrica.

\section{Abstract}

This research aims at building an instrument to evaluate the linguistic-discursive quality of disciplinary texts produced by law students. A rubric for evaluating writing across the law curriculum was developed using a qualitative study with a phenomenological design. The interviews with teachers used to elaborate the instrument allowed to collect the linguis-

$1 \quad$ La investigación fue realizada gracias al financiamiento del Proyecto FONDECYT Nº11170128, titulado "Caracterización discursiva de géneros producidos por estudiantes de Derecho y Medicina: su relación con la percepción de la autoeficacia en la escritura, la calidad general del texto y la evaluación disciplinar", y al apoyo de la Dirección de Investigación de la Universidad de La Serena. 
tic-discursive properties that specialized texts should have and the criteria that disciplinary teachers should evaluate in such texts. Once validated through different procedures, the rubric was composed by sixteen dimensions and four macrodimensions, i.e., communicative situation, communication of legal knowledge, global and sentence structure and regulations. The final version of the instrument obtained a considerable agreement in both the content validity $(K=0.73)$ and the intercoder reliability $(K=0,62)$. This validated instrument would be of great value for the different actors involved in the process of forming lawyers for at least two reasons: on the one hand, more precise information regarding the acquisition of the legal competence across the law curriculum could be obtained; on the other, intervention strategies adapted to the needs of the students and tools for developing a didactic of writing in law can be elaborated.

Keywords: academic literacy; writing evaluation; law; rubric. 


\section{Introducción}

En la formación de cualquier profesional, las habilidades comunicativas, en general, y la escritura, en particular, tienen un rol fundamental. Más concretamente, la producción de textos escritos de calidad representa un problema central para estudiantes y profesores, pues su falta de dominio constituye una de las principales causas de reprobación, retraso y deserción de una parte importante de los estudiantes de educación superior (Cristina, 2010; Uribe y Carrillo, 2014; Meza y González-Catalán, 2020). Por ello, la enseñanza y evaluación de la escritura son actividades esenciales en cualquier disciplina, máxime en Derecho, área en la que el buen dominio de las habilidades comunicativas es imprescindible; sobre todo de las habilidades de escritura, que, según Kosse y ButleRitchie (2003), son vitales para la práctica de la ley. En esta línea, es sabido que la calidad de la retroalimentación formativa oportuna es un elemento central en la enseñanza y evaluación de la escritura (Knight y otros, 2018). Sin embargo, los docentes, con formación en ciencias jurídicas, pero no en didáctica ni en evaluación de la escritura, muchas veces carecen de las herramientas adecuadas para evaluar los aspectos lingüísticos y discursivos de los textos que producen sus estudiantes.

En esta investigación abordamos la evaluación de la calidad de textos disciplinares producidos por estudiantes de Derecho, línea de estudio que cuenta con algunos datos relevantes, pero poco sistematizados. A pesar de ello, los trabajos revisados coinciden en las carencias de los estudiantes al escribir un texto de calidad (Tapia y otros, 2003; Kolb y otros, 2013; Graham y otros, 2016; Wilson y otros, 2017). Por esto, diversas investigaciones, similares a la nuestra, han presentado propuestas para evaluar aspectos lingüistico-discursivos de textos producidos por estudiantes en la educación superior. Si bien ellas constituyen un avance en el área, aún persisten una serie de vacíos. Primero, se han propuesto instrumentos para evaluar solo un género concreto (Hart y Breland, 1994; Tapia y otros, 2002, 2003; Morales, 2004). Segundo, no se han considerado los actores del proceso de evaluación de textos especializados, sino que las propuestas se basan en lo que un experto, en lengua o en Derecho, señala que debe evaluarse en términos lingüístico-discursivos (Tapia y otros, 2002, 2003; Morales, 2004; Gidi y Weihofen, 2018). Tercero, se han diseñado instrumentos de evaluación de la escritura genéricos, sin considerar el área de especialización de los estudiantes (Sundeen, 2014; Evensen y otros, 2016). Cuarto, en general, los instrumentos no han sido validados (Tapia y otros, 2002, 2003; Morales, 2004). Quinto, la evaluación es realizada en situaciones simuladas de escritura (Neira y Ferreira, 2011). Sexto, se han presentado instrumentos de evaluación que prometen evaluar la escritura en Derecho, cuando, en realidad, se evalúa la respuesta escrita a preguntas de comprensión (Cabrera, 2014). En síntesis, tal como señala Hartig (2016: 66), "although the body of research on legal language is significant, analysis of the kinds of texts that lawyers learn to write in the vocational stage of their training remains limited".

En este marco, nuestro objetivo es proponer un instrumento que supere dichos vacíos y permita evaluar las características lingüístico-discursivas de los textos que producen los estu- 
diantes de Derecho en su formación y que deberán generar durante su desempeño profesional. Esto, considerando la visión de los miembros de la comunidad disciplinar, que son quienes, en definitiva, deben evaluar a los estudiantes, pero no cuentan con las herramientas necesarias para hacerlo.

\section{Instrumentos de evaluación de la escritura}

En este apartado, presentamos algunos antecedentes teóricos sobre la evaluación de la escritura en términos generales y también en el ámbito disciplinar específico de Derecho. En Chile, contamos con algunos avances iniciales como, por ejemplo, una pauta de evaluación para los aspectos más relevantes de un informe universitario (Tapia y otros, 2003). Según las autoras, el instrumento corresponde a una escala de apreciación, cuyos 31 indicadores se agrupan en 9 dimensiones (título, introducción, desarrollo, procedimientos, objetivos e hipótesis, resultados o presentación de datos, conclusiones, referencias bibliográficas y calidad general del escrito). Cada indicador se evalúa según 4 categorías de valoración asociadas con sus respectivos puntajes. Tal como ellas reconocen, su instrumento no ha sido validado, aunque en su práctica docente resultó eficaz (Tapia y otros, 2002). A pesar de su aporte, este trabajo ha sido de tipo más bien exploratorio, con una muestra pequeña de sujetos, y se ha centrado en un género predeterminado y transversal a distintas áreas disciplinares.

Otra propuesta es la de Morales (2004), quien, de manera similar a nuestra investigación, destina su trabajo, especialmente, a los docentes no especialistas en lengua, y entrega algunos lineamientos sobre qué elementos evaluar de los textos de sus estudiantes. Los tipos de textos sobre cuya evaluación reflexiona son, principalmente, los que se suelen englobar bajo el término general de monografía, esto es, ensayos escolares, informes de investigación bibliográfica, etc. En este marco, el autor propone las siguientes categorías que, desde su punto de vista, deberían revisarse en cualquier escrito: grafía, vocabulario, gramática y cohesión, estructura (Morales, 2004). A nuestro juicio, el problema de este trabajo es que ha sido realizado desde una perspectiva normativa, sin una investigación empírica de sustento y a partir, exclusivamente, del conocimiento de su autor, quien ha señalado arbitrariamente qué evaluar de la escritura de los estudiantes y cómo hacerlo.

En el ámbito del Derecho, encontramos el instrumento CODICE-Derecho (Cabrera, 2014), cuyo objetivo es medir la comprensión de textos y la escritura de los estudiantes de primer año de la carrera de Derecho. Concretamente, el apartado dedicado a la escritura consiste en la construcción de una respuesta a una pregunta de comprensión. Así, 3 de los 5 textos jurídicos que componen el instrumento van acompañados de una pregunta abierta, que "propone una tarea de escritura en torno a un género específico (por ejemplo: texto argumentativo, definición, caracterización), basándose en temas tratados en el texto estímulo. Por ello, el estudiante debe haber leído comprensivamente el fragmento para elaborar una buena respuesta” (Cabrera, 2014: 4). Tal como señala la autora, la corrección de estos textos (o respues- 
tas, más específicamente) se realiza mediante rúbricas, que consideran aspectos tales como: planteamiento de ideas, cohesión oracional y normas ortográficas, cada uno con un puntaje determinado y 5 niveles de desempeño.

Claramente, CODICE-Derecho tiene la fortaleza de vincular las habilidades comunicativas a la disciplina concreta, pues los textos provienen de manuales y revistas especializadas del área; y, además, su selección se realizaría en conjunto con los profesores de Derecho (Cabrera, 2014). Sin embargo, a nuestro juicio, el foco del instrumento no está en la producción de textos, pues ella se utiliza exclusivamente como herramienta para responder tres preguntas de desarrollo sobre los textos presentados como estímulo, por lo que, en ningún caso, se evalúa la producción de textos disciplinares. Además, no se entregan mayores detalles sobre la rúbrica utilizada para evaluar las respuestas desarrolladas.

También en el ámbito jurídico, Hart y Breland (1994) trabajaron en la identificación de los elementos esenciales de una buena escritura legal. Para ello, se centraron en el análisis de un género disciplinar específico, a saber, 237 memorandos legales (legal memoranda), producidos por estudiantes de Derecho de primer semestre. Para identificar dichos elementos consideraron los juicios de: profesores de Derecho, especialistas en evaluación en humanidades y dos asesores jurídicos. A pesar de algunos desacuerdos durante el proceso de análisis, los autores lograron determinar los elementos más importantes de los memorandos legales y el peso relativo de cada uno en la evaluación de los profesores. Concretamente, identificaron 53 elementos, con los que construyeron una taxonomía con aquellos más evaluados (positiva o negativamente) por profesores de escritura jurídica (instructors of legal writing). Dichos elementos, y su frecuencia, son: 1. Emisión e identificación de hechos clave (81,4\%), 2. Citación (uso y forma) (69\%), 3. Adecuación a los detalles (64\%), 4. Expresiones legales y contexto (63,7\%), 5. Relevancia de los hechos declarados (63,3\%), 6. Aplicación de la ley a los hechos (63\%), 7. Transición, flujo y continuidad lógica (61,8\%), 8. Descripción y comprensión de la autoridad (58,6\%), 9. Analogía y comparación de los hechos (58,4\%), 10. Claridad (57,3\%). Como observamos, solo algunos de los elementos se relacionan con la calidad lingüístico-discursiva de los textos, pues la mayoría de ellos son, más bien, propios del género o del conocimiento disciplinar.

Un trabajo similar al anterior es realizado por Gidi y Weihofen (2018), quienes describieron algunos principios centrales de la escritura legal. Según los autores, no se trataría de reglas inmutables, sino más bien de directrices dependientes del contexto, del objetivo y del propio gusto y criterio del escritor. Para reforzar esta idea los autores señalan: "Take all these principles into consideration and you may write effectively; follow them blindly and your style will become formulaic" (Gidi y Weihofen, 2018: v). Concretamente, para los autores mencionados las recomendaciones para una buena escritura legal giran en torno a los siguientes puntos: precisión, concisión, sencillez, claridad, fuerza y organización. De forma similar al trabajo de Morales (2004), la propuesta de Gidi y Weihofen (2018) también resulta arbitraria, en el sentido 
de que no se establecen criterios para definir por qué considerar esos elementos y no otros, aunque tiene el valor de avocarse al ámbito concreto de la escritura jurídica. Por otro lado, se centra en recomendaciones para la producción de textos, no para su evaluación.

Si bien los trabajos presentados representan un avance en la evaluación de la escritura, y un punto de partida para esta investigación, ninguno de ellos reporta una indagación empírica previa, que permita asegurar la adaptación total del instrumento a la disciplina o género en que será aplicado. En nuestro caso, los procedimientos de indagación previa son: a) aplicación de un cuestionario en línea y uno en vivo a docentes de Derecho, que permitió saber qué profesores trabajan la escritura con sus estudiantes y, además, conocer el repertorio exacto de los géneros que ellos producen; b) entrevistas a los docentes que realizan tareas de escritura disciplinar en sus clases, cuyas respuestas guiaron los elementos que debían conformar nuestro instrumento. Todos estos procedimientos son detallados, a continuación, en el apartado dedicado a los aspectos metodológicos.

\section{Métodos}

Este estudio se enmarca en un paradigma interpretativo, pues la generación del instrumento nace de la comprensión que tienen los docentes de la escritura en Derecho y, en particular, de las propiedades que debe poseer un escrito perteneciente a este campo del saber. En este sentido, el proceso investigativo ha considerado la concepción que los abogados tienen, como sujetos informantes, acerca de una realidad, la que en este caso corresponde a la calidad lingüístico-discursiva de sus textos disciplinares. Desde esta mirada, los discursos emitidos por los informantes acerca del fenómeno estudiado han sido interpretados de manera tal que logre prevalecer el valor otorgado por estos y podamos develar el significado de aquello que no es evidente (Bautista, 2011). Desde esta perspectiva, intentamos esclarecer e identificar las propiedades que, a juicio de los docentes disciplinares de la carrera de Derecho, debe contener un texto que responde a parámetros de calidad lingüístico-discursiva en el ámbito jurídico.

Posteriormente, con dicha información generamos un instrumento que permita evaluar el desempeño, en términos lingüístico-discursivos, de los abogados en formación a la hora de producir un texto disciplinar, esto es, propio de su profesión. Para cumplir con los propósitos del estudio, hemos optado por un diseño fenomenológico, pues el instrumento generado ha surgido a partir de las experiencias humanas relativas a un fenómeno (Creswell, 2009). En este caso, dicho fenómeno corresponde la calidad lingüístico-discursiva de un texto en el ámbito del Derecho, de acuerdo con los requerimientos que los propios docentes disciplinares han declarado.

En relación con la muestra, trabajamos con un muestreo intencional, pues la selección de los participantes ha respondido a las intenciones propias del estudio. La modalidad específica ha sido el muestreo opinático, ya que la selección de los informantes ha obedecido a un criterio 
estratégico (Ruiz, 2012), asociado al cumplimiento de requerimientos tales como la integración de actividades de escritura dentro de cátedras disciplinares impartidas por los docentes participantes. Esta información fue obtenida mediante dos procedimientos complementarios: cuestionario en línea y cuestionario en vivo a todos los docentes de las escuelas de Derecho que contemplamos en esta investigación. Esto nos permitió seleccionar a los docentes a entrevistar, utilizando como criterio de inclusión la realización de actividades de producción de textos propios de la labor de un abogado en los cursos que dictan. Así, entrevistamos a 5 abogados, todos docentes en escuelas de Derecho de universidades pertenecientes al CRUCH (3 en una institución estatal y 2 en una no estatal).

La información sobre las características de los textos producidos durante la formación de abogados la obtuvimos mediante la técnica de entrevista en profundidad, la que nos permitió, a través de la interacción entrevistado-entrevistador, construir el significado otorgado al fenómeno estudiado. En este sentido, el proceso de intercambio entre los participantes de esta instancia ha sido no estructurado, utilizando preguntas con respuesta abierta orientadas a la comprensión de una realidad sin categorías de respuesta preestablecidas (Ruiz, 2012). Las temáticas de la entrevista surgen a partir de los vacíos que reconocimos en investigaciones previas sobre evaluación de textos disciplinares (Hart y Breland, 1994; Cabrera, 2014; Gidi y Weihofen, 2018) y, luego, fueron ajustadas a partir de distintos procesos de validación.

Así, las preguntas diseñadas para la entrevista fueron validadas, primero, a través de un proceso de triangulación y, segundo, de la realización de una entrevista piloto para probar las preguntas. En la triangulación, las preguntas fueron revisadas por distintos expertos: un abogado, una lingüista, un estudiante de periodismo y una profesora de castellano. Tras el análisis, se realizaron los ajustes y se evaluaron nuevamente las preguntas. El proceso se repitió hasta despejar cualquier incertidumbre. Por otra parte, para el pilotaje, con las preguntas ya revisadas, entrevistamos a un abogado-docente, a fin de probar las preguntas y la realización de la entrevista en general.

En relación con el análisis de la información, este se realizó de forma manual. Para ello, aplicamos la técnica de análisis de contenido, entendiéndola como el proceso de interpretación de los datos recolectados a través de su reducción y categorización (Bautista, 2011). Esta técnica la trabajamos sobre los discursos de los docentes, logrando dejar de manifiesto aquel sentido latente de las representaciones construidas acerca del fenómeno. Al respecto, Krippendorff (1980) señala que esta técnica tiene un carácter objetivo, sistemático y cualitativo, que permite la formulación de inferencias exhaustivas, reproducibles y válidas que pueden aplicarse a un contexto (Porta y Silva, 2003). A partir de ello, pudimos levantar una serie de categorías, correspondientes a las propiedades que debe poseer un texto que responde a los requerimientos de calidad lingüístico-discursiva definidos por la comunidad disciplinar y la norma jurídica. Esto, posteriormente, nos ha llevado a generar la propuesta de instrumento de evaluación, en particular una rúbrica, considerando macrodimensiones, dimensiones y 
descriptores, de acuerdo con lo señalado por los especialistas. El modo como el contenido de las entrevistas corresponde a las categorías del instrumento se detalla en la sección siguiente, pues ellas pertenecen, en definitiva, a los resultados de este trabajo. Este proceso se ejemplifica, además, en la figura 2.

Una vez diseñada nuestra rúbrica, la sometimos a tres procedimientos distintos de validación: a) revisión por parte de una experta, b) validación de contenido por jueces expertos y c) pilotaje; estos dos últimos fueron realizados en dos etapas. En el primer procedimiento mencionado, la rúbrica fue revisada por una investigadora extranjera experta en escritura académica y en evaluación de la competencia discursiva. Tras esta primera revisión, se realizaron los ajustes pertinentes.

En la segunda etapa aludida, llevamos a cabo un proceso de validación de contenido por parte de jueces expertos. Concretamente, enviamos el instrumento a 5 jueces chilenos y extranjeros con distintos perfiles: un profesor de escritura en una carrera de Derecho, un experto en evaluación, un abogado-docente, un lingüista experto en escritura académica y un psicólogo; todos ellos hablantes nativos de español y con, al menos, un postgrado en su ámbito de especialización. En paralelo, realizamos una primera aplicación piloto del instrumento diseñado, que permitió evaluar el grado de acuerdo o consistencia entre distintos anotadores. Concretamente, 5 anotadores aplicaron la rúbrica a un mismo texto producido por un estudiante de Derecho, recogido en una de las instituciones que son parte de esta investigación. El perfil de los anotadores también fue variado: un abogado-docente (distinto del evaluador del juicio de expertos), dos estudiantes de Derecho de último año, una profesora de castellano y un estudiante de periodismo que se ha desempeñado como ayudante en cursos de redacción general.

Luego de estos dos procedimientos de validación (juicio de expertos y pilotaje), realizamos los ajustes pertinentes al instrumento, obteniendo, así, una versión mejorada de la rúbrica. Tanto a los jueces expertos como a los anotadores del pilotaje les solicitamos un registro escrito de sus observaciones, las que fueron consideradas en los ajustes que realizamos al instrumento luego de esta primera etapa de validación.

La versión modificada de la rúbrica se sometió nuevamente, en una segunda etapa, a juicio de expertos y pilotaje, para lo que recurrimos a 3 nuevos jueces chilenos y extranjeros, todos hablantes nativos de español y con al menos un postgrado en su ámbito de especialización. El perfil específico fue: una abogada-docente, una profesora de escritura en Derecho y un lingüista experto en escritura académica. Mientras que para el pilotaje trabajamos con tres anotadores: una profesora de castellano y dos estudiantes de Derecho de último año.

Para calcular el acuerdo entre jueces expertos como también entre anotadores utilizamos el porcentaje de acuerdo y el coeficiente Kappa de Fleiss (1971). El objetivo de este último es determinar el grado de acuerdo entre tres o más evaluadores mediante la exclusión de 
las concordancias que se puedan atribuir al azar. Para realizar todos los cálculos utilizamos ReCal3: Intercoder reliability calculation (Freelon, 2010). Todos los datos referentes a los índices numéricos obtenidos de estos procedimientos de validación se detallan en el apartado siguiente, pues, en definitiva, son resultados de la aplicación del instrumento propuesto.

Los procedimientos metodológicos se sintetizan, a continuación, en la figura 1:

\section{FIGURA 1}

Síntesis de procedimientos metodológicos

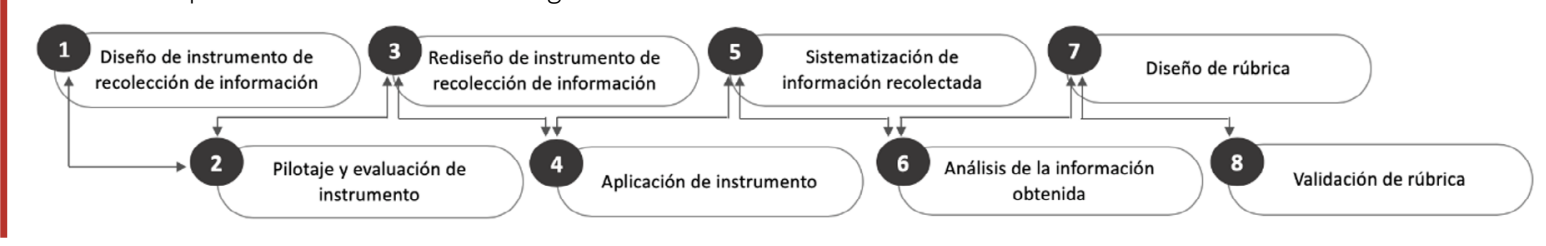

\section{Análisis y discusión de resultados}

Los resultados de nuestra investigación dicen relación, básicamente, con la presentación del instrumento que proponemos. Para ello, seguimos un criterio cronológico, esto es, desde los resultados que nos permiten confirmar en la práctica el vacío identificado hasta aquellos que dicen relación con la primera aplicación o pilotaje del instrumento. Esto, así como también los aspectos que los entrevistados han declarado como necesarios de fortalecer en los abogados en formación, se podrá visualizar en los segmentos que integramos y que forman parte de las entrevistas sostenidas para este estudio.

El proceso de generación del instrumento orientado a evaluar la calidad lingüístico-discursiva de los textos producidos en la formación de abogados obedece a la necesidad de fortalecer esta competencia en dichos profesionales. En este sentido, y de acuerdo con lo mencionado por los especialistas entrevistados, nuestro primer hallazgo es la confirmación de que, si bien existe la creencia de que el ejercicio jurídico de un profesional del Derecho es eminentemente oral, quienes forman parte de esta comunidad académica y disciplinar afirman que esto no necesariamente es así y que la escritura siempre ha sido una habilidad indispensable para el trabajo jurídico. Ejemplo de ello es el siguiente fragmento:

En este minuto, bueno, eh, se acostumbra y hay una especie de percepción de que el trabajo del abogado, hoy día, está derivando hacia lo estrictamente oral [...]. Y esa es una percepción parcialmente correcta, solo parcialmente ¿Por qué?, eh, porque los procedimientos, incluso los procedimientos reformados, tanto en el área penal como en familia y laboral, eh, sigue teniendo una base escrita desde la presentación de la demanda, como es la contestación y la tramitación de los escritos propios, ¿no es cierto?, de las etapas procesales. Se sigue necesitando de la escritura como herramienta de comunicación entre el operador jurídico y el tribunal. Eso es así [...] [DULS_I]. 
De acuerdo con esto, y en concordancia con lo señalado por Kosse y ButleRitchie (2003), se hace cada día más necesario contar con herramientas que permitan fortalecer la competencia escritural de quienes se encuentran en proceso de formación como abogados. Esto, dada la relevancia que tiene la producción escrita en el campo jurídico, pues los profesionales del área deben generar sus escritos, procurando respetar las exigencias de la comunidad discursiva en la que se encuentran. No obstante, se transforma en una tarea compleja, pues no cuentan con las habilidades básicas para ello, lo que los lleva, incluso, a exponer su calidad profesional, considerando que son variados los lectores del texto generado, tal como se evidencia a continuación:

Por lo tanto, claro, en la medida que uno no tiene habilidades eh, de una buena redacción, bueno, ortografía que es como elemental, uno queda en evidencia. De hecho, me ha tocado ver cómo a mi litigante, ahora no como profe, que los jueces le llaman la atención a los colegas cuando no entienden la demanda, por ejemplo. “¿Sabe qué? Aclare esto, no se entiende” [DULS_1].

En esta línea, según lo señalado por los profesores entrevistados, las debilidades asociadas a la competencia escritural en los abogados en proceso de formación son variadas y van desde aspectos básicos, asociados a la norma, hasta problemas referidos a la utilización correcta de categorías gramaticales específicas en una construcción oracional. Estos elementos básicos de la escritura parecen ser fundamentales en la producción de textos en el ámbito jurídico, pues una investigación reciente ha evidenciado que los aspectos gramaticales y normativos están presentes, incluso, entre los elementos que los abogados en ejercicio destacan como propios de la escritura jurídica clara (Meza y otros, 2020). Producto de ello es que se hace necesario contar con espacios que permitan reforzar, y evaluar eficientemente, la producción escrita en los estudiantes, de tal manera que sea posible superar aquellos errores que cometen al momento de responder a tareas de escritura propias de la disciplina. Un ejemplo es el siguiente:

El desconocimiento de la estructura de la oración a mí a veces me sorprende. Y de la básica, o sea, sujeto, verbo y predicado [...] Mira, yo trabajé con este curso desde que eran mechones, desde que entraron el primer semestre del año pasado, y traen deficiencias del colegio. Traen, o sea, no saber reconocer la correcta utilización de las reglas de puntuación ¿ya? (el uso de la coma, el punto y coma, el punto seguido, el punto aparte, etc.), la correcta utilización de la mayúscula, la correcta utilización de las categorías gramaticales; o sea, por ejemplo, qué rol tiene un adverbio, qué rol tiene un artículo, el artículo definido, indefinido, etc. Entonces, esas carencias, cuando se ven enfrentados al momento de redactar un ensayo, por ejemplo, que es mucha idea personal, se pierden [DUCN_2].

Desde nuestro punto de vista, una las de las estrategias posibles para fortalecer esta área es la evaluación, pero no entendida exclusivamente como aquel proceso asociado a calificar el desempeño de un estudiante, sino como una oportunidad de aprendizaje. Esto significa, a nuestro juicio, que la acción de evaluar el trabajo de los estudiantes debe transformarse en una instancia de reconocimiento de los errores frecuentes y, a partir de ello, entregar orien- 
taciones para la mejora y superación. Pese a que este proceso es una forma de lograr mejorar la competencia escritural en los abogados en formación, no se trata de una práctica que se desarrolle de manera frecuente, por lo que evidenciamos un claro vacío formativo en una habilidad que es requerimiento para el desempeño en las actividades académicas y profesionales, como se advierte en la cita siguiente:

En algunas partes donde me ha tocado trabajar, sí, eh, se está, tímidamente, iniciando el, digamos, desarrollo de rúbricas para las evaluaciones orales, pero no, no me he encontrado yo con que me han facilitado o entregado, ¿no es cierto?, alguna rúbrica o pauta de cotejo, como sea, respecto a la habilidad de escritura. No cuento, no tengo [DULS_1].

Es por lo anterior que, creemos, se hace necesario reorientar el proceso de evaluación de la escritura en el campo de la formación de abogados, de tal manera que, a través de ello, se les brinde una oportunidad para desarrollar y fortalecer la habilidad de producción escrita. En este sentido, cuando nos referimos al proceso de evaluación de la escritura, buscamos establecer una comparación entre un producto escrito que ha sido construido por un individuo, en circunstancias específicas, y determinados estándares o referentes que han sido definidos intencionalmente a partir de una serie de requerimientos dispuestos por una comunidad disciplinar. Por tanto, en nuestra concepción, evaluar la producción escrita no implica únicamente el reconocimiento de la presencia o ausencia de ciertas propiedades, sino que va más allá de ello, pues lo que esperamos es visualizar en qué medida el escrito elaborado se adecua a las exigencias de la comunidad discursiva. Desde esta perspectiva, concebimos la evaluación de la escritura como un proceso que permite verificar el grado de adecuación de un texto a los requerimientos establecidos, en primera instancia, por la actividad en la que se enmarca la producción escrita y, en segunda, por las normas definidas por el campo de conocimiento. Al asumir el proceso de evaluación desde un enfoque cualitativo, lo que nos interesa es definir las cualidades que debe poseer un texto jurídico para efectos de ser un discurso adecuado en el campo jurídico.

De acuerdo con lo anterior, para desarrollar un proceso de evaluación como el que nos interesa requerimos determinar cuáles son esas cualidades que finalmente se transformarán en nuestros estándares al momento de definir en qué medida un texto se adecua o no a circunstancias específicas. En concreto, los participantes de la comunidad han declarado que las propiedades asociadas a la calidad de un texto de Derecho están relacionadas con dar respuesta a los requerimientos exigidos por el campo disciplinar, así como también por la norma jurídica. Por tanto, la producción de textos escritos se posiciona como una capacidad necesaria que todo sujeto que desea insertarse en el área en cuestión debe adquirir, a fin de poder responder satisfactoriamente a cada una de las actividades propias del ámbito. Esta habilidad se asume como un proceso complejo en el que interactúan distintos factores, por lo que el hecho de observar la calidad lingüístico-discursiva de un texto en Derecho implica la consideración de cada uno de ellos. 
Los propios miembros de la comunidad, en las entrevistas, han declarado una serie de atributos que deben ser considerados al momento de implementar un proceso de evaluación de un texto. Los distintos aspectos señalados por los especialistas entrevistados los hemos analizado y sistematizado, como se ejemplifica en la figura 2. Así vemos cómo cada fragmento dado por los entrevistados nos ha guiado en la determinación de los elementos que incorporamos en nuestra rúbrica:

\section{FIGURA 2}

Ejemplo análisis de entrevistas para construcción de rúbrica

"[...] un párrafo bien escrito uno supone que esté arquitectónicamente bien equilibrado. Yo siempre le digo a mis estudiantes que una página soporta entre veinte y treinta líneas y esas veinte o treinta líneas debiesen estar estructuradas a lo más en tres párrafos, que cada párrafo, idealmente, debiese estar separado por un punto aparte, debiese cada párrafo no tener no más de dos puntos seguidos y dentro de esos puntos, además de las frases principales y subordinadas, debiese estar estructurada con sujeto, verbo y predicado [... $]^{\prime \prime}$
Organización textual en párrafos

Construcción de oraciones

Este tipo de análisis nos permitió diseñar una rúbrica, que denominamos RECLiTeD (Rúbrica

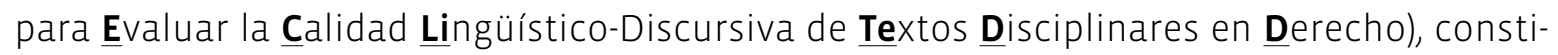
tuida por distintas dimensiones y macrodimensiones. Las dimensiones corresponden a las características lingüístico-discursivas específicas representativas de los textos de calidad mencionadas por los entrevistados, por lo que, en consecuencia, equivalen a los descriptores a evaluar en nuestra rúbrica. Mientras que las macrodimensiones corresponden a una agrupación que, a partir de nuestra experiencia, hemos hecho de las distintas dimensiones. En la figura 3 presentamos presentamos gráficamente una síntesis de nuestro instrumento.

La figura 3 se origina a partir de la información que hemos levantado, analizado y sistematizado a partir de las entrevistas realizadas, por lo que constituye una síntesis de RECLiTeD. Las distintas dimensiones identificadas las agrupamos en las siguientes macrodimensiones: a) situación comunicativa, b) comunicación del conocimiento jurídico, c) estructura global y oracional, y d) normativa. A continuación, definimos cada una de estas macrodimensiones, de acuerdo con lo declarado por los abogados que imparten docencia en las universidades estudiadas y, también, a partir de nuestro propio conocimiento sobre el tema. Posteriormente, para cada dimensión planteamos distintos niveles de desempeño para construir nuestra rúbrica. En adelante, para mayor claridad, ejemplificamos la descripción de las macrodimensiones con fragmentos de las entrevistas realizadas, que son los que, finalmente, justifican la incorporación a la rúbrica de cada dimensión y macrodimensión.

a) Situación comunicativa: conjunto de requerimientos textuales asociados al contexto en el que se enmarca la actividad de escritura. Esto implica la producción de un texto adecuado al tema, vale decir, aborda la materia definida en el marco de la tarea de escritura; al propósito, esto significa que el texto cumple con la finalidad propia del género discursivo o de la instan- 


\section{FIGURA 3}

Síntesis dimensiones y macrodimensiones del instrumento propuesto

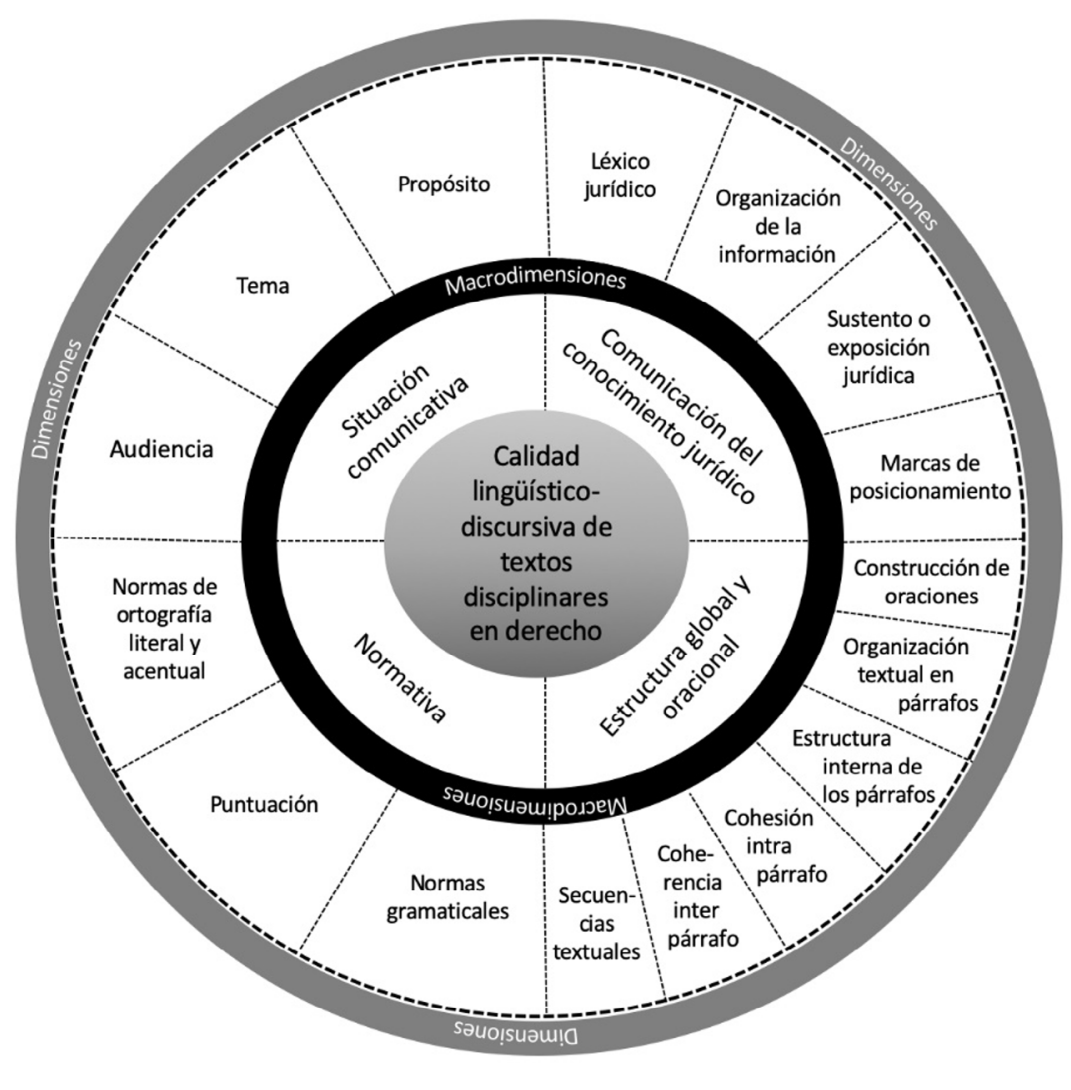

cia comunicativa en la que se desarrolla la escritura; y a las características de la audiencia, es decir, que responde a las necesidades de los potenciales lectores del texto. Esto se advierte en la siguiente afirmación:

[...] A pesar de que el cliente no necesariamente sea abogado, lo que él quiere es una opinión jurídica, entonces no hay que bajar el lenguaje ni tampoco omitir el lenguaje técnico [...] [DUCN_I].

b) Comunicación del conocimiento jurídico: propiedades de un texto jurídico definidas por la comunidad disciplinar. Entre estas se reconocen la utilización de léxico jurídico, que significa emplear terminología técnica de manera adecuada a los requerimientos de la situación comunicativa; organización de la información, que implica ordenar la información contenida en el texto utilizando criterios adecuados a los requerimientos del género; sustento o exposición jurídica, esto es, en el primer caso (sustento), fundamentar la tesis u opinión jurídica en argumentos de hecho y Derecho que son consistentes con los requerimientos de la situación, y, en el segundo (exposición), describir de manera precisa la situación jurídica en cuestión, incorporando la documentación necesaria; y marcas de posicionamiento, que dice relación con la inclusión de marcas lingüísticas que evidencian el rol del escritor en 
el contexto de la comunicación jurídica y el dominio del conocimiento especializado. Un fragmento que justifica estos elementos es:

[...] lo que me interesa es si son capaces de lograr percibir y, por lo tanto, transmitir de manera escrita cuál es el problema jurídico que se plantea y, a su vez, si logran ver si ese problema jurídico se ve en nuestra legislación, y que sean capaces de hacer las conexiones necesarias con la legislación que se investiga [...] [DULS_3].

c) Estructura global y oracional: atributos del texto jurídico referidos a la organización textual en párrafos, construcción de oraciones, estructura interna de los párrafos, cohesión intrapárrafo, coherencia interpárrafos y secuencias textuales. En particular, el primero de estos elementos se orienta a la elaboración de párrafos conformados por oraciones identificables y cuya extensión permite un desarrollo claro y completo de las ideas integradas en el párrafo; el segundo alude a la construcción de oraciones constituidas por un referente claramente identificable y dos o tres ideas que lo nutren; el tercero refiere a la generación de párrafos a partir de una oración temática, la cual se nutre de oraciones de apoyo que ayudan a hacer progresar la información; el cuarto alude al empleo de diversos mecanismos de cohesión para establecer relaciones explícitas y coherentes entre los diversos segmentos que componen los párrafos; el quinto está orientado al establecimiento de relaciones coherentes, lográndose evidenciar un hilo conductor temático (de manera implícita o explícita) y progresión temática, vinculando la información conocida con aquella que es nueva; y el sexto recoge la utilización de secuencias textuales adecuadas en el desarrollo del texto, las que permiten responder a todos los requerimientos de la situación comunicativa. Dos fragmentos de las entrevistas que sirven de ejemplo son los siguientes:

[...] un párrafo bien escrito uno supone que esté arquitectónicamente bien equilibrado. Yo siempre le digo a mis estudiantes que una página soporta entre veinte y treinta líneas, y esas veinte o treinta líneas debiesen estar estructuradas a lo más en tres párrafos, que cada párrafo, idealmente, debiese estar separado por un punto aparte, debiese cada párrafo no tener no más de dos puntos seguidos y dentro de esos puntos, además de las frases principales y subordinadas, debiese estar estructurada con sujeto, verbo y predicado [...] [DULS_2]

[...] El estudiante, muchas veces, ¿qué hace? tiene la idea y la vuelca completamente y después se olvida de revisarla, ver si el párrafo que escribió tiene conexión con el que viene o no lo tiene ¿ah? El alumno solo piensa y lo vuelca todo sin una ordenación lógica [...] [DULS_3].

d) Normativa: conjunto de propiedades, asociadas al correcto uso de la lengua española, con las que debe contar el texto jurídico. En particular, estas corresponden a normas gramaticales, referidas a la construcción oracional donde se evidencia coherencia entre las distintas categorías gramaticales; normas de ortografía acentual y literal, que refiere al correcto uso de tildes y de letras; y puntuación, que es la utilización adecuada de variados signos de puntuación. Un fragmento de las entrevistas que sustenta estas dimensiones es el siguiente: 
[...] Entonces, la ... el cómo se escribe es sumamente importante en el desempeño profesional. Incluso, no tan solo el cómo y el respecto a las reglas ortográficas y gramaticales, sino que visualmente y la forma del texto [...] [DUCN_I].

En síntesis, toda la información recogida, analizada y sistematizada a partir de las entrevistas realizadas a los docentes de las escuelas de Derecho que son parte de esta investigación nos ha permitido diseñar una rúbrica para evaluar la calidad lingüístico-discursiva de los textos disciplinares propios de la labor de un abogado, producidos por estudiantes de Derecho en formación. Luego de todos los procesos de validación mencionados en el apartado metodológico, RECLiTeD ha quedado constituida por 4 macrodimensiones, 16 dimensiones asociadas a ellas (ver figura 3) y distintos niveles de desempeño para cada una de estas dimensiones. Por razones de espacio, en este artículo ofrecemos solo los elementos constituyentes de la rúbrica propuesta; sin embargo, una versión completa de ella se puede encontrar en el siguiente vínculo: https://paulinameza.org/instrumentos-de-evaluacion/. Además, en la figura 4 presentamos un ejemplo de la dimensión "Léxico jurídico" del instrumento. En ella podemos observar los niveles de progresión para dicha dimensión y el puntaje asociado a cada uno de ellos:

\section{FIGURA 4}

Dimensión “Léxico jurídico” de RECLiTeD

\begin{tabular}{|c|c|c|c|c|}
\hline \multirow{2}{*}{$\begin{array}{l}\text { 4. Léxico } \\
\text { jurídico }\end{array}$} & o PUNTO & 2 PUNTOS & 4 PUNTOS & 6 PUNTOS \\
\hline & $\begin{array}{l}\text { Integra de manera } \\
\text { adecuada la } \\
\text { terminología técnica } \\
\text { de la disciplina, } \\
\text { incorporando } \\
\text { los conceptos } \\
\text { especializados en } \\
\text { construcciones que } \\
\text { permiten comunicar } \\
\text { su significado, sin } \\
\text { embargo, presenta } \\
\text { cinco o más errores } \\
\text { en el texto. }\end{array}$ & $\begin{array}{l}\text { Integra de manera } \\
\text { adecuada la } \\
\text { terminología técnica } \\
\text { de la disciplina, } \\
\text { incorporando } \\
\text { los conceptos } \\
\text { especializados en } \\
\text { construcciones que } \\
\text { permiten comunicar } \\
\text { su significado, sin } \\
\text { embargo, presenta } \\
\text { tres o cuatro } \\
\text { errores en el texto. }\end{array}$ & $\begin{array}{l}\text { Integra de manera } \\
\text { adecuada la } \\
\text { terminología técnica } \\
\text { de la disciplina, } \\
\text { incorporando } \\
\text { los conceptos } \\
\text { especializados en } \\
\text { construcciones que } \\
\text { permiten comunicar } \\
\text { su significado, sin } \\
\text { embargo, presenta } \\
\text { uno o dos errores } \\
\text { en el texto. }\end{array}$ & $\begin{array}{l}\text { Integra de manera } \\
\text { adecuada, en } \\
\text { todo el texto, la } \\
\text { terminología técnica } \\
\text { de la disciplina, } \\
\text { incorporando } \\
\text { los conceptos } \\
\text { especializados en } \\
\text { construcciones que } \\
\text { permiten comunicar } \\
\text { su significado. }\end{array}$ \\
\hline
\end{tabular}

La constitución de la rúbrica presentada, y sistematizada en la figura 3, evidencia que los elementos para la evaluación de la escritura propuestos previamente desde una perspectiva normativa y arbitraria, en su mayoría, no coinciden con los que realmente se requieren en una comunidad disciplinar específica. Así, por ejemplo, no sería pertinente evaluar en textos de abogados en formación ninguno de los puntos señalados por Tapia y otros (2002, 2003), salvo la calidad general del escrito, criterio que es muy poco específico en su propuesta. Tampoco los elementos establecidos por Morales (2004) son pertinentes para textos de Derecho. Ello, por cierto, podría explicarse porque los autores mencionados están pensando en géneros académicos transversales. 
En el caso de Cabrera (2014), si bien su propuesta se ubica en el ámbito del Derecho, los aspectos a evaluar son muy generales y no apuntan a todo lo que, en nuestro trabajo, los entrevistados han indicado como características de calidad de los textos que solicitan a sus estudiantes. Además de los problemas ya señalados en el apartado teórico de este artículo.

Con Hart y Breland (1994), por otro lado, que también se centran en un género jurídico, coincidimos solo en algunos de los puntos detectados por ellos, a saber: "expresiones legales", "transición, flujo y continuidad lógica". Esto podría deberse a que los autores señalados se centran exclusivamente en el género memorando legal. Además, no se focalizan solo en aspectos lingüísticos, sino también en aquellos que tienen que ver con el contenido de los textos (relevancia de los hechos declarados, por ejemplo), que no es nuestro foco de interés.

En el caso de Gidi y Weihofen (2018), las dimensiones de nuestra rúbrica coinciden con dos de sus puntos: precisión y organización. Dichos autores incluyen la sencillez entre sus criterios textuales; sin embargo, según lo recogido en nuestras entrevistas, los estudiantes y abogados siempre deben pensar en una audiencia especializada, por lo que la sencillez no constituye, necesariamente, un criterio de calidad lingüístico-discursiva. Nuevamente, las diferencias encontradas podrían explicarse por la indagación previa en una comunidad específica que sustenta nuestro trabajo. En este sentido, creemos que las ideas planteadas en los párrafos anteriores refuerzan la importancia de realizar una investigación empírica previa al diseño de cualquier intervención en la didáctica o evaluación de la escritura, de modo de responder a las necesidades concretas de una comunidad disciplinar específica.

Como señalamos previamente, una vez diseñada la rúbrica, ella pasó por distintas etapas de validación, cuyos resultados indicamos a continuación:

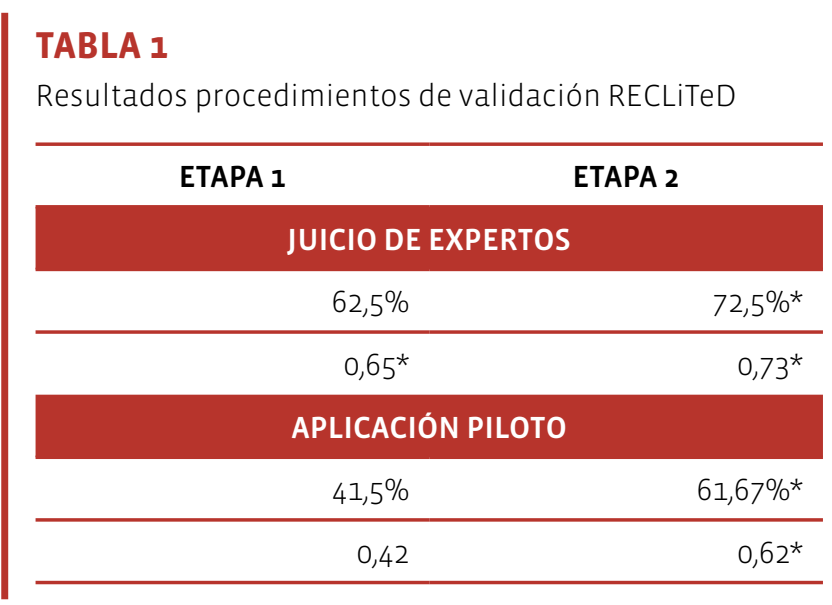

De acuerdo con la tabla 1 , en la primera etapa, en el juicio de expertos obtuvimos un porcentaje de acuerdo que no alcanza el valor de referencia mínimo para la validación establecido por Blessing y Chakrabarti (2009), mientras que el resultado de Kappa de Fleiss (1971) alcanzó un 
valor que corresponde a un acuerdo considerable (Landis y Koch, 1977). Por su parte, para la aplicación piloto también obtuvimos un porcentaje de acuerdo insuficiente y un coeficiente Kappa de Fleiss (1971) moderado (Landis y Koch, 1977).

Para la segunda etapa del proceso de validación por jueces, el porcentaje de acuerdo superó el valor de referencia mínimo establecido por Blessing y Chakrabarti (2009), mientras que el coeficiente Kappa de Fleiss (1971) alcanzó un acuerdo considerable (Landis y Koch, 1977). En tanto que, en el segundo pilotaje, el coeficiente Kappa de Fleiss (1971) indicó un acuerdo considerable. En consecuencia, en la segunda aplicación piloto y de juicio de expertos, todos los índices mejoraron, lo que indica que, sin duda, los ajustes realizados optimizaron el instrumento. Además, estos índices nos permiten afirmar que el instrumento propuesto alcanza un índice de validez adecuado.

\section{Conclusiones}

En primer lugar, a partir de los resultados obtenidos, podemos afirmar que nuestra propuesta viene a cubrir un vacío reconocido por los miembros de la comunidad disciplinar investigada, quienes, si bien asumen la importancia de los aspectos lingüísticos y discursivos en su ámbito, admiten también las falencias de sus estudiantes en esta área, y agregan a ello el hecho de no tener formación en evaluación y tampoco contar con instrumentos adecuados para evaluar la calidad lingüístico-discursiva de los textos producidos por los abogados en formación. En esta misma línea, logramos cubrir todos los vacíos planteados inicialmente en la introducción de este trabajo.

Uno de los valores de RECLiTeD radica en el hecho de que no se ha construido desde una perspectiva normativa ni arbitraria, esto es, sobre la base de lo que nosotros como especialistas en escritura creemos y decidimos que se debe evaluar en los textos de los abogados en formación. Por el contrario, se trata de una rúbrica empíricamente fundada, esto es, son los propios profesores de las carreras de Derecho incluidas en esta investigación quienes nos han señalado qué evalúan ellos y cuáles son, en definitiva, las características de calidad de los escritos producidos en su comunidad disciplinar. En esta misma línea, el instrumento no surge a partir de situaciones simuladas de escritura (Neira y Ferreira, 2011), sino que tiene su origen en la identificación de los géneros, y sus características, que realmente producen los estudiantes de Derecho durante su formación y que, además, deberán producir en su desempeño profesional.

Otro valor de la rúbrica que proponemos radica en su versatilidad, pues no ha sido diseñada para evaluar un único género predeterminado, sino que permite valorar un abanico amplio de textos disciplinares del ámbito del Derecho, propios de la labor de un abogado. Esta versatilidad se evidencia también en el doble objetivo que permite cumplir la rúbrica diseñada: uno práctico y uno didáctico. Por una parte, el propósito práctico es evaluar la calidad 
lingüístico-discursiva de los textos que componen el corpus del proyecto mayor en el que se enmarca esta investigación. El objetivo didáctico, por otra parte, es brindar un instrumento de evaluación a profesores de Derecho que requieran evaluar las características lingüísticodiscursivas de los textos que producen sus estudiantes, o, en el polo contrario, a profesores de habilidades comunicativas que requieran evaluar textos disciplinares en Derecho. Al respecto, es importante precisar que en ningún caso pretendemos evaluar con esta rúbrica aspectos de contenido de los textos disciplinares.

La versatilidad del instrumento se evidencia también en las múltiples audiencias y usos que podría tener. Por una parte, puede ser útil a profesores que ejercen docencia en Derecho, cumpliendo, de esta forma, un fin académico, pues les ayudaría a determinar cuáles y cómo evaluar aspectos lingüístico-discursivos en los textos de sus estudiantes, sobre la base de dimensiones declaradas y validadas por su comunidad. Por otra parte, el RECLiTeD puede resultar útil también para aquellas personas que se están insertando en el ámbito del Derecho, pues les permitirá conocer cuáles son los requerimientos lingüístico-discursivos en su área. En este sentido, el instrumento, además, puede resultar orientador para la didáctica y la evaluación de la escritura en Derecho. Así, por ejemplo, un profesor podría trabajar en su clase un determinado género, utilizando el nivel de desempeño más alto de la rúbrica para guiar a sus estudiantes en su producción textual.

Por otro lado, RECLiTeD es un instrumento de origen multidisciplinario, pues, si bien ha sido diseñado por dos investigadores y docentes del ámbito de la evaluación y de la escritura académica, la descripción de las necesidades y de las características de un texto de calidad provienen de las declaraciones de los abogados que forman estudiantes en Derecho. Luego, la validación se llevó a cabo por parte de especialistas de diversas áreas: Derecho, Psicología, Lingüística, etc.; con diferentes grados de inserción disciplinar (pregrado, magíster, doctorado, etc.) y de distintas instituciones de educación superior chilenas y extranjeras.

Creemos que contar con un instrumento aceptado y bien valorado será de gran utilidad para los distintos actores involucrados en el proceso de formación de abogados, pues permitirá obtener información más precisa respecto del nivel de adquisición de la competencia escritural en Derecho. Así también, su uso permitirá definir estrategias de intervención adecuadas a las necesidades de los estudiantes y proveer herramientas que posibiliten el desarrollo de una didáctica de la escritura en Derecho. Dado lo anterior es que la rúbrica generada se posiciona como un instrumento flexible, útil para la disciplina en general e independiente de variables como el género, nivel en la carrera o asignatura en que se desee utilizar. Esta flexibilidad se evidencia también en que sus dimensiones pueden ser aplicadas de manera independiente y de acuerdo con las necesidades que tenga el docente al momento de implementar una práctica pedagógica orientada al fortalecimiento de la producción escrita. De este modo se trata de un instrumento versátil que puede ser aplicado con diversas intencionalidades evaluativas (diagnóstica, formativa o sumativa) y en 
distintos momentos (proceso o final), pues todo dependerá de las decisiones pedagógicas y evaluativas que tome el docente como mediador en el proceso de adquisición de la habilidad de producción escrita en el campo jurídico.

Asimismo, la rúbrica se posiciona como una herramienta orientadora para la didáctica de la escritura en Derecho, al presentar una descripción de lo que se espera que el estudiante sea capaz de demostrar a través de su escritura. En este sentido, proporciona información al docente respecto de microhabilidades que deben ser trabajadas, aspectos lingüístico-discursivos relevantes de considerar en las actividades que conforman la estrategia pedagógica que se implementa en la formación de abogados y la definición de elementos que deben ser foco de interés al momento de generar los procesos de retroalimentación al trabajo realizado por los estudiantes.

La necesidad de contar con instrumentos precisos de evaluación de la escritura en Derecho ha sido avalada por autores como Kosse y ButleRitchie (2003), quienes han señalado que, si fuera posible identificar un conjunto de estándares, podríamos contar con una medida consistente para evaluar la redacción de los graduados en Derecho. A ello agregan que una herramienta de evaluación de este tipo ayudaría a los profesores de redacción legal a reforzar las habilidades típicas de producción de textos. Esto es, precisamente, lo que pretendemos con RECLITeD.

\section{Bibliografía citada}

BAutista, Nelly, 2011: Proceso de la investigación cualitativa. Epistemología, metodología y aplicaciones, Bogotá: Editorial El Manual Moderno.

BLESSING, Lucienne, y Amaresh ChaKRABARTI, 2009: DRM, a Design Research Methodology, Dordrecht: Springer.

Cabrera, Marcela, 2014: "El instrumento CODICE-Derecho y su aplicación en la Facultad de Derecho de la Universidad de Chile”, Revista Pedagogía Universitaria y Didáctica del Derecho 1, 2-19.

Creswell, John, 2009: Research design: qualitative, quantitative and mixed methods approaches, Londres: Sage.

CRISTINA, Inés, 2010: La deserción universitaria en primer año. Trabajo Final de Grado, Universidad Nacional de La Plata en La Plata, Argentina.

EVEnSEN, Lars, y otros, 2016: "Standards as a tool for teaching and assessing cross-curricular writing", The Curriculum Journal 27, 229-245. 
FLEISS, Joseph, 1971: "Measuring Nominal Scale Agreement among many Rater”, Psychological Bulletin 76, 378-382.

Freelon, Deen, 2010: "ReCal: Intercoder reliability calculation as a web service", International Journal of Internet Science 5, 20-33.

Graham, Steve, y otros, 2016: "Assessing the writing achievement of young struggling writers: application of generalizability theory", Learning Disability Quarterly 39, 72-82.

Gidı, Antonio, y Henry WelHofen, 2018: Legal writing style, Minnesota: West Academic Publishing.

HART, Frederick, y Hunter BRELAND, 1994: "Defining Legal Writing: An Empirical Analysis of the Legal Memorandum" [https://digitalrepository.unm.edu/law_facultyscholarship/142, fecha de consulta: 25 de junio de 2018].

HARTIG, Alissa, 2016: “Conceptual blending in legal writing: Linking definitions to facts”, English for Specific Purposes 42, 66-75.

KNIGHT, Simon, y otros, 2018: "Designing academic writing analytics for civil law student selfassessment", International Journal of Artificial Intelligence in Education 28, 1-28.

Kolb, Kenneth, Kyle Longest y Mollie Jensen, 2013: "Assessing the Writing Process Do WritingIntensive First-Year Seminars Change How Students Write?”, Teaching Sociology 41, 20-31.

Kosse, Susan, y David Butleritchie, 2003: "How Judges, Practitioners, and Legal Writing Teachers Assess the Writing Skills of New Law Graduates: A Comparative Study", Journal of Legal Education 53, 80-102.

KrippendorfF, Klaus, 1980: Content analysis an introduction to its Methodology, London: Sage.

LANDIS, Richard, y Gary KocH, 1977: "The measurement of observer agreement for categorical data", Biometrics 33, 159-174.

Meza, Paulina, y Felipe Gonzalez-Catalán, 2020: "Evaluación de la calidad lingüístico-discursiva en textos disciplinares: propuesta de un instrumento analítico para valorar la producción escrita en la formación de médicos”, Logos: Revista de Lingüística, Filosofía y Literatura 30, 3-17.

Meza, Paulina, Felipe Gonzalez-Catalan, Carmen López-Ferrero e Israel Gutiérrez, 2020: “Plain writing in the legal field: An approach from the discourse of specialists", Discourse Studies 22, 356-383.

Morales, Francisco, 2004: “Evaluar la escritura, sí... Pero ¿Qué y cómo evaluar?”, Acción Pedagógica 13, 38-49. 
Neira, Angie, y Anita Ferreira, 2011: "Escritura académica: un modelo metodológico efectivo basado en tareas y enfoque cooperativo”, Literatura y Lingüística 24, 143-159.

PorTA, Luis y Miriam Silva, 2003: "La investigación cualitativa: el análisis de contenido en la investigación educativa” [http://www.uccor.edu.ar/paginas/reduc/porta.pdf, fecha de consulta: 26 de junio de 2018].

Ruiz, José, 2012: Metodología de la investigación cualitativa, Bilbao: Universidad de Deusto.

SUndEEn, Todd, 2014: "Instructional rubrics: Effects of presentation options on writing quality", Assessing Writing 21, 74-88.

TAPIA, Mónica, Beatriz Arancibia y Gina BuRdiles, 2002: "Criterios para la evaluación de informes académicos de estudiantes universitarios”, REXE: Revista de Estudios y Experiencias en Educación 1, 117-126.

TAPIA, Mónica, Gina Burdiles y Beatriz Arancibia, 2003: "Aplicación de una pauta diseñada para evaluar informes académicos universitarios”, Revista Signos. Estudios de Lingüística 36, 249-257.

URIBE, Olga, y Stefany CARRILlo, 2014: "Relación entre la lecto-escritura, el desempeño académico y la deserción estudiantil”, Entramado 10 (2), 272-285.

WiLson, Frances, Simon ChILd e Irenka Suto, 2017: "Assessing the transition between school and university: Differences in assessment between A level and university in English", Arts and Humanities in Higher Education 162, 188-208. 